Acta Crystallographica Section E

Structure Reports

Online

ISSN 1600-5368

\title{
Potassium morpholine-4-carbodithioate monohydrate. Corrigendum
}

\section{Ana C. Mafud ${ }^{a *}$ and Maria Teresa Prado Gambardella ${ }^{b}$}

a Instituto de Física de São Carlos, Universidade de São Paulo, Av. Trabalhador

Sãocarlense, 400, Caixa Postal 369, 13566-590 São Carlos, SP, Brazil, and ' Instituto de Química de São Carlos, Universidade de São Paulo, Av. Trabalhador Sãocarlense, 400, Caixa Postal 369, 13566-590 São Carlos, SP, Brazil

Correspondence e-mail: mafud@usp.br

Received 2 April 2013; accepted 2 April 2013

The author list in the paper by Mafud [Acta Cryst. (2012), E68, $\mathrm{m} 1025$ ] is corrected.

In the paper by Mafud (2012), one of the original authors was omitted. The correct list is given above.

\section{References}

Mafud, A. C. (2012). Acta Cryst. E68, m1025. 
Acta Crystallographica Section E

\section{Structure Reports}

Online

ISSN 1600-5368

\section{Potassium morpholine-4-carbodithioate monohydrate}

\author{
Ana C. Mafud \\ Instituto de Física de São Carlos, Universidade de São Paulo, Av. Trabalhador \\ Sãocarlense, 400, Caixa Postal 369, 13566-590 São Carlos, SP, Brazil \\ Correspondence e-mail: mafud@usp.br
}

Received 30 May 2012; accepted 28 June 2012

Key indicators: single-crystal X-ray study; $T=290 \mathrm{~K}$; mean $\sigma(\mathrm{C}-\mathrm{C})=0.005 \AA$; $R$ factor $=0.047 ; w R$ factor $=0.130 ;$ data-to-parameter ratio $=24.7$.

In the ionic title compound, $\mathrm{K}^{+} \cdot \mathrm{C}_{5} \mathrm{H}_{8} \mathrm{NOS}_{2}{ }^{-} \cdot \mathrm{H}_{2} \mathrm{O}$, the morpholine ring of the morpholine-4-carbodithioate anion has a chair conformation. The potassium cation is coordinated by four $\mathrm{S}$ and four $\mathrm{O}$ atoms in a bipyramidal reversed geometry. In the crystal, the three components are linked, generating infinite two-dimensional networks that lie parallel to the $b c$ plane. These layers are linked via $\mathrm{O}-\mathrm{H} \cdots \mathrm{S}$ hydrogen bonds, forming a three-dimensional structure.

\section{Related literature}

For the crystal structures of similar compounds, see: Oskarsson et al. (1979); Albertsson et al. (1980); Ymén (1982); Mafud \& Gambardella (2011a,b); Mafud et al. (2011). For puckering parameters, see: Cremer \& Pople (1975).

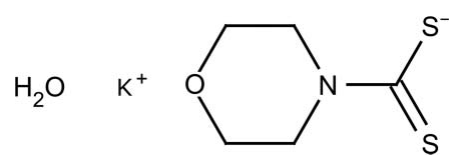

\section{Experimental}

Crystal data

$\mathrm{K}^{+} \cdot \mathrm{C}_{5} \mathrm{H}_{8} \mathrm{NOS}_{2}{ }^{-} \cdot \mathrm{H}_{2} \mathrm{O}$

$M_{r}=219.36$

Monoclinic, $P 2_{1} / c$

$a=6.7235(10) \AA$

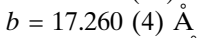

$c=8.1904$ (10) $\AA$

$\beta=108.994(10)^{\circ}$

Data collection

Enraf-Nonius TurboCAD-4 diffractometer

Absorption correction: refined from $\Delta F$ (Walker \& Stuart, 1983)

$T_{\text {min }}=0.512, T_{\max }=0.818$

2779 measured reflections

$$
\begin{aligned}
& V=898.7(3) \mathrm{A}^{3} \\
& Z=4 \\
& \text { Mo K } \alpha \text { radiation } \\
& \mu=1.01 \mathrm{~mm}^{-1} \\
& T=290 \mathrm{~K} \\
& 0.45 \times 0.30 \times 0.20 \mathrm{~mm}
\end{aligned}
$$

\footnotetext{
2618 independent reflections 1615 reflections with $I>2 \sigma(I)$ $R_{\text {int }}=0.027$

3 standard reflections every $120 \mathrm{~min}$ intensity decay: $10 \%$
}

\section{Refinement}

$R\left[F^{2}>2 \sigma\left(F^{2}\right)\right]=0.047$

$w R\left(F^{2}\right)=0.130$

$S=1.01$

2618 reflections

106 parameters

3 restraints

Table 1

Hydrogen-bond geometry $\left(\AA{ }^{\circ}\right)$.

\begin{tabular}{lllll}
\hline$D-\mathrm{H} \cdots A$ & $D-\mathrm{H}$ & $\mathrm{H} \cdots A$ & $D \cdots A$ & $D-\mathrm{H} \cdots A$ \\
\hline $\mathrm{O} 2-\mathrm{H} 1 O \cdots \mathrm{S}^{\mathrm{i}}$ & $0.86(4)$ & $2.45(4)$ & $3.219(3)$ & $149(3)$ \\
$\mathrm{O} 2-\mathrm{H} 2 O \cdots \mathrm{S}^{\mathrm{ii}}$ & $0.85(3)$ & $2.87(5)$ & $3.462(3)$ & $129(4)$ \\
\hline
\end{tabular}

Symmetry codes: (i) $-x-1, y+\frac{1}{2},-z+\frac{1}{2}$; (ii) $-x-1,-y,-z+1$.

Data collection: CAD-4 EXPRESS (Enraf-Nonius, 1994); cell refinement: CAD-4 EXPRESS; data reduction: XCAD4 (Harms \& Wocadlo, 1995); program(s) used to solve structure: SIR92 (Altomare et al., 1994); program(s) used to refine structure: SHELXL97 (Sheldrick, 2008); molecular graphics: ORTEP-3 for Windows (Farrugia, 1997); software used to prepare material for publication: Win $G X$ (Farrugia, 1999).

The author is grateful to the CNPq, National Counsel of Technological and Scientific Development, for supporting this study.

Supplementary data and figures for this paper are available from the IUCr electronic archives (Reference: SU2446).

\section{References}

Albertsson, J., Oskarsson, Å., Ståhl, K., Svensson, C. \& Ymén, I. (1980). Acta Cryst. B36, 3072-3078.

Altomare, A., Cascarano, G., Giacovazzo, C., Guagliardi, A., Burla, M. C., Polidori, G. \& Camalli, M. (1994). J. Appl. Cryst. $27,435$.

Cremer, D. \& Pople, J. A. (1975). J. Am. Chem. Soc. 97, 1354-1358.

Enraf-Nonius (1994). CAD-4 EXPRESS. Enraf-Nonius, Delft, The Netherlands.

Farrugia, L. J. (1997). J. Appl. Cryst. 30, 565

Farrugia, L. J. (1999). J. Appl. Cryst. 32, 837-838.

Harms, K. \& Wocadlo, S. (1995). XCAD4. University of Marburg, Germany.

Mafud, A. C. \& Gambardella, M. T. P. (2011a). Acta Cryst. E67, m942.

Mafud, A. C. \& Gambardella, M. T. P. (2011b). Acta Cryst. E67, o879.

Mafud, A. C., Sanches, E. A. \& Gambardella, M. T. (2011). Acta Cryst. E67, o2008.

Oskarsson, A., Ståhl, K., Svensson, C. \& Ymen, I. (1979). Eur. Cryst. Meet, 5, 67.

Sheldrick, G. M. (2008). Acta Cryst. A64, 112-122.

Walker, N. \& Stuart, D. (1983). Acta Cryst. A39, 158-166.

Ymén, I. (1982). Acta Cryst. B38, 2671-2674. refinement $\Delta \rho_{\min }=-0.61$ e $\AA^{-3}$ independent and constrained $\Delta \rho_{\max }=0.50 \mathrm{e}^{-3}$ 


\section{supporting information}

Acta Cryst. (2012). E68, m1025 [https://doi.org/10.1107/S1600536812029613]

\section{Potassium morpholine-4-carbodithioate monohydrate}

\section{Ana C. Mafud}

\section{S1. Comment}

The title compound, Fig. 1, is composed of a morpholinedithiocarbamate anion in contact with a potassium cation, which in turn is linked with a water molecule of crystallization. The crystal structure of similar compounds, for example Sodium 1-R-carbodithioate dihydrate, have been reported (Oskarsson et al., 1979; Albertsson et al., 1980; Ymén, 1982; Mafud \& Gambardella et al., 2011a,b).

The six-membered morpholine ring has a chair conformation with Puckering parameters [Cremer \& Pople, 1975)] Q = $0.548(3) \AA, \theta=173,3(3)^{\circ}, \varphi 2=2,6(3,4)^{\circ}$.

In the crystal, a polymeric structure is built by coordination of the potassium cation to four sulfur $[\mathrm{K} \cdots \mathrm{S}=3.2670(13)$ 3.3797 (14) $\AA]$ and four oxygen $[\mathrm{K} \cdots \mathrm{O}=2.828$ (3) - 3.007 (3) $\AA]$ atoms, with a bi-pyramidal reversed geometry. This configuration generates close packed layers which remain cohesive in crystal stacking by van der Waals interactions. The distances of these contacts are slightly less than the sum of the van der Waals radii.

The crystal packing gives rise to a supramolecular structure, whose infinite two-dimensional network lies parallel to the

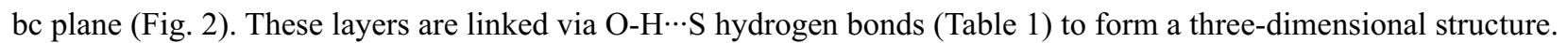

\section{S2. Experimental}

The potassium salts of DTC were prepared by direct reaction between amine and carbon disulfide $\left(\mathrm{CS}_{2}\right)$ in the presence of a stoichiometric amount of potassium hydroxide in ethanol/water 1:1 $(v: v)$. The reaction mixture was placed in the freezer for $12 \mathrm{~h}$ and then filtered through a Büchner funnel, washed with cold ether and the product recrystallized in an ethanol-water mixture 1:1 (v:v). The obtained solid was recrystallized from ethanol-water 1:1 $(v / v)$ and dried in a vacuum oven at $323 \mathrm{~K}$ for $8 \mathrm{~h}$. Colourless crystals, suitable for X-ray diffraction analysis, were obtained. On heating they sublimed and decomposed.

\section{S3. Refinement}

The H-atom positions of the water molecule were located in a difference Fourier map and were refined with $\mathrm{U}_{\text {iso }}(\mathrm{H})=$ $1.5 \mathrm{U}_{\mathrm{eq}}(\mathrm{O}) ; \mathrm{O}-\mathrm{H}=0.86(4)$ and 0.85 (3) $\AA$. The C-bound H-atoms of the anion were included in calculated positions and treated as riding atoms: $\mathrm{C}-\mathrm{H}=0.97 \AA$, with $U_{\text {iso }}(\mathrm{H})=1.2 U_{\text {eq }}$ (parent C-atom). 


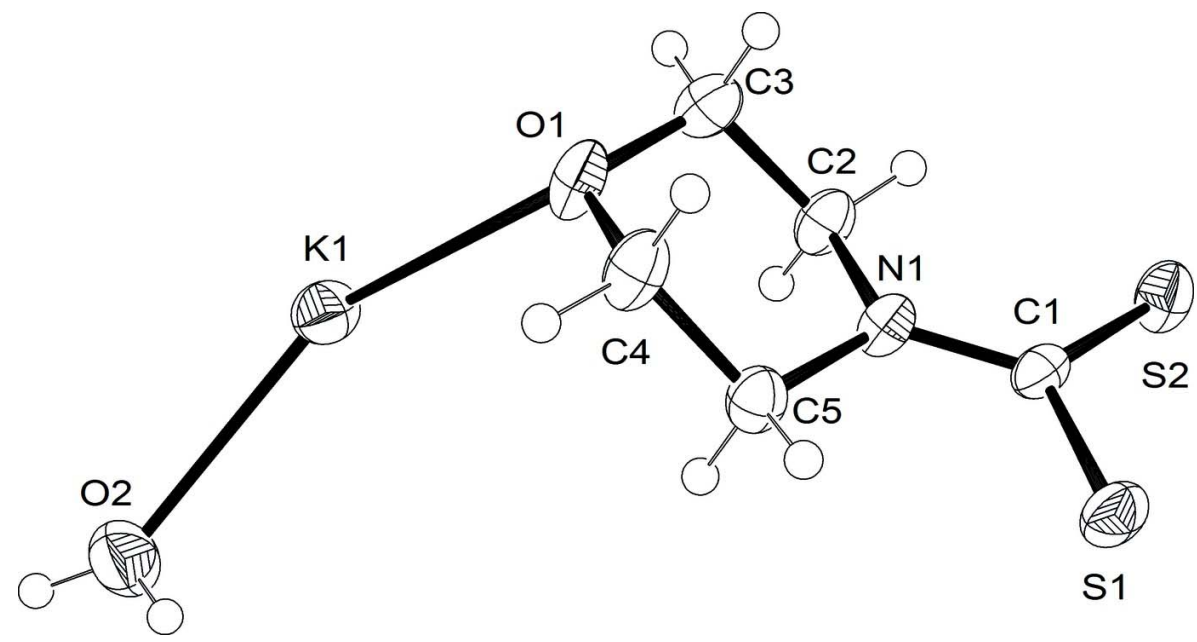

Figure 1

A view of the molecular structure of the asymmetric unit of the title compound, with the numbering scheme. The displacement ellipsoids are drawn at the $50 \%$ probability level.

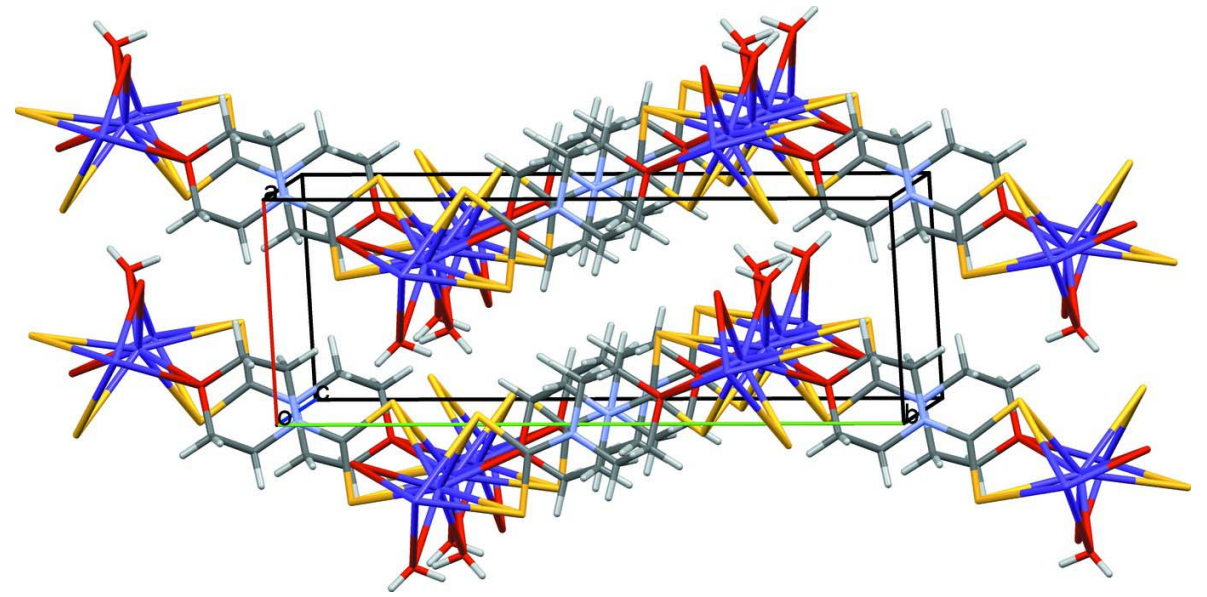

Figure 2

The view along the $\mathrm{c}$ axis of the crystal packing of the title compound.

Potassium morpholine-4-carbodithioate monohydrate

Crystal data

$\mathrm{K}^{+} \cdot \mathrm{C}_{5} \mathrm{H}_{8} \mathrm{NOS}_{2}^{-} \cdot \mathrm{H}_{2} \mathrm{O}$

$F(000)=456$

$M_{r}=219.36$

Monoclinic, $P 2{ }_{1} / c$

$D_{\mathrm{x}}=1.621 \mathrm{Mg} \mathrm{m}^{-3}$

Hall symbol: -P 2ybc

Mo $K \alpha$ radiation, $\lambda=0.71073 \AA$

$a=6.7235$ (10) $\AA$

Cell parameters from 16 reflections

$b=17.260(4) \AA$

$c=8.1904(10) \AA$

$\beta=108.994(10)^{\circ}$

$\theta=9.8-18.3^{\circ}$

$\mu=1.01 \mathrm{~mm}^{-1}$

$T=290 \mathrm{~K}$

$V=898.7(3) \AA^{3}$

Prism, colourless

$Z=4$

$0.45 \times 0.3 \times 0.2 \mathrm{~mm}$ 


\section{Data collection}

Enraf-Nonius TurboCAD-4 diffractometer

Radiation source: Enraf Nonius FR590

Graphite monochromator

non-profiled $\omega / 2 \theta$ scans

Absorption correction: part of the refinement model $(\Delta F)$ (Walker \& Stuart, 1983)

$T_{\min }=0.512, T_{\max }=0.818$

2779 measured reflections

\section{Refinement}

Refinement on $F^{2}$

Least-squares matrix: full

$R\left[F^{2}>2 \sigma\left(F^{2}\right)\right]=0.047$

$w R\left(F^{2}\right)=0.130$

$S=1.01$

2618 reflections

106 parameters

3 restraints

Primary atom site location: structure-invariant direct methods
2618 independent reflections

1615 reflections with $I>2 \sigma(I)$

$R_{\text {int }}=0.027$

$\theta_{\text {max }}=30.0^{\circ}, \theta_{\min }=2.4^{\circ}$

$h=-9 \rightarrow 8$

$k=0 \rightarrow 24$

$l=0 \rightarrow 11$

3 standard reflections every $120 \mathrm{~min}$ intensity decay: $10 \%$
Secondary atom site location: difference Fourier map

Hydrogen site location: inferred from neighbouring sites

$\mathrm{H}$ atoms treated by a mixture of independent and constrained refinement

$w=1 /\left[\sigma^{2}\left(F_{\mathrm{o}}^{2}\right)+(0.0644 P)^{2}\right]$

where $P=\left(F_{\mathrm{o}}^{2}+2 F_{\mathrm{c}}^{2}\right) / 3$

$(\Delta / \sigma)_{\max }<0.001$

$\Delta \rho_{\max }=0.50 \mathrm{e} \AA^{-3}$

$\Delta \rho_{\min }=-0.61$ e $\AA^{-3}$

Special details

Geometry. Bond distances, angles etc. have been calculated using the rounded fractional coordinates. All su's are estimated from the variances of the (full) variance-covariance matrix. The cell esds are taken into account in the estimation of distances, angles and torsion angles

Refinement. Refinement of $F^{2}$ against ALL reflections. The weighted $R$-factor $w R$ and goodness of fit $S$ are based on $F^{2}$, conventional $R$-factors $R$ are based on $F$, with $F$ set to zero for negative $F^{2}$. The threshold expression of $F^{2}>\sigma\left(F^{2}\right)$ is used only for calculating $R$-factors(gt) etc. and is not relevant to the choice of reflections for refinement. $R$-factors based on $F^{2}$ are statistically about twice as large as those based on $F$, and $R$ - factors based on ALL data will be even larger.

Fractional atomic coordinates and isotropic or equivalent isotropic displacement parameters $\left(\AA^{2}\right)$

\begin{tabular}{lllll}
\hline & $x$ & $y$ & $z$ & $U_{\text {iso }} * / U_{\text {eq }}$ \\
\hline K1 & $-0.28085(12)$ & $0.24637(4)$ & $0.48217(9)$ & $0.0366(2)$ \\
S1 & $-0.06278(13)$ & $-0.16683(4)$ & $0.69255(11)$ & $0.0320(2)$ \\
S2 & $0.36659(13)$ & $-0.09853(4)$ & $0.80129(12)$ & $0.0336(3)$ \\
O1 & $-0.1501(4)$ & $0.13219(12)$ & $0.7743(3)$ & $0.0366(7)$ \\
O2 & $-0.5955(4)$ & $0.27515(16)$ & $0.1617(4)$ & $0.0496(9)$ \\
N1 & $0.0172(4)$ & $-0.01514(13)$ & $0.7295(3)$ & $0.0261(7)$ \\
C1 & $0.1003(5)$ & $-0.08662(15)$ & $0.7399(4)$ & $0.0240(8)$ \\
C2 & $0.1460(5)$ & $0.05521(16)$ & $0.7583(4)$ & $0.0293(9)$ \\
C3 & $0.0660(5)$ & $0.11438(18)$ & $0.8574(4)$ & $0.0334(10)$ \\
C4 & $-0.2719(5)$ & $0.06303(18)$ & $0.7624(5)$ & $0.0348(10)$ \\
C5 & $-0.2096(4)$ & $0.00131(17)$ & $0.6581(4)$ & $0.0289(8)$ \\
$\mathrm{H} 10$ & $-0.682(6)$ & $0.3070(18)$ & $0.093(5)$ & $0.0740^{*}$ \\
$\mathrm{H} 2 \mathrm{~A}$ & 0.14220 & 0.07690 & 0.64810 & $0.0350^{*}$ \\
$\mathrm{H} 2 \mathrm{~B}$ & 0.29080 & 0.04230 & 0.82250 & $0.0350^{*}$ \\
$\mathrm{H} 2 \mathrm{O}$ & $-0.645(7)$ & $0.2294(12)$ & $0.154(6)$ & $0.0740^{*}$
\end{tabular}




\begin{tabular}{|c|c|c|c|c|}
\hline $\mathrm{H} 3 \mathrm{~A}$ & 0.08450 & 0.09460 & 0.97230 & $0.0400^{*}$ \\
\hline H3B & 0.14850 & 0.16140 & 0.86940 & $0.0400 *$ \\
\hline $\mathrm{H} 4 \mathrm{~A}$ & -0.41980 & 0.07520 & 0.70910 & $0.0420 *$ \\
\hline H4B & -0.25230 & 0.04350 & 0.87770 & $0.0420 *$ \\
\hline H5A & -0.28850 & -0.04570 & 0.65890 & $0.0350 *$ \\
\hline H5B & -0.24380 & 0.01850 & 0.53940 & $0.0350 *$ \\
\hline
\end{tabular}

Atomic displacement parameters $\left(\AA^{2}\right)$

\begin{tabular}{lllllll}
\hline & $U^{11}$ & $U^{22}$ & $U^{33}$ & $U^{12}$ & $U^{13}$ & $U^{23}$ \\
\hline $\mathrm{K} 1$ & $0.0408(4)$ & $0.0356(4)$ & $0.0341(4)$ & $-0.0006(3)$ & $0.0130(3)$ & $-0.0026(3)$ \\
$\mathrm{S} 1$ & $0.0383(4)$ & $0.0207(3)$ & $0.0366(4)$ & $-0.0060(3)$ & $0.0116(3)$ & $-0.0032(3)$ \\
$\mathrm{S} 2$ & $0.0297(4)$ & $0.0270(4)$ & $0.0454(5)$ & $0.0033(3)$ & $0.0141(3)$ & $-0.0004(3)$ \\
$\mathrm{O} 1$ & $0.0370(12)$ & $0.0220(10)$ & $0.0551(15)$ & $0.0040(9)$ & $0.0210(11)$ & $-0.0031(10)$ \\
$\mathrm{O} 2$ & $0.0444(15)$ & $0.0393(14)$ & $0.0546(17)$ & $0.0006(12)$ & $0.0018(13)$ & $0.0048(13)$ \\
$\mathrm{N} 1$ & $0.0270(12)$ & $0.0204(11)$ & $0.0339(14)$ & $-0.0009(9)$ & $0.0142(10)$ & $-0.0035(10)$ \\
$\mathrm{C} 1$ & $0.0313(14)$ & $0.0203(13)$ & $0.0231(14)$ & $-0.0011(11)$ & $0.0124(11)$ & $-0.0031(11)$ \\
$\mathrm{C} 2$ & $0.0310(15)$ & $0.0205(13)$ & $0.0400(18)$ & $-0.0044(11)$ & $0.0164(14)$ & $-0.0021(12)$ \\
$\mathrm{C} 3$ & $0.0356(17)$ & $0.0278(15)$ & $0.0381(18)$ & $-0.0040(12)$ & $0.0140(14)$ & $-0.0072(13)$ \\
$\mathrm{C} 4$ & $0.0326(16)$ & $0.0267(15)$ & $0.051(2)$ & $0.0004(12)$ & $0.0218(15)$ & $-0.0025(14)$ \\
$\mathrm{C} 5$ & $0.0267(14)$ & $0.0255(14)$ & $0.0349(16)$ & $-0.0005(11)$ & $0.0108(13)$ & $-0.0004(12)$
\end{tabular}

Geometric parameters (A, $\stackrel{\circ}{)}$

\begin{tabular}{|c|c|c|c|}
\hline $\mathrm{K} 1-\mathrm{O} 1$ & $3.002(2)$ & $\mathrm{N} 1-\mathrm{C} 2$ & $1.465(4)$ \\
\hline $\mathrm{K} 1-\mathrm{O} 2$ & $2.828(3)$ & $\mathrm{N} 1-\mathrm{C} 1$ & $1.346(4)$ \\
\hline $\mathrm{K} 1-\mathrm{S} 1^{\mathrm{i}}$ & $3.2670(13)$ & $\mathrm{N} 1-\mathrm{C} 5$ & $1.472(4)$ \\
\hline $\mathrm{K} 1-\mathrm{S} 2^{\mathrm{i}}$ & $3.3630(13)$ & $\mathrm{C} 2-\mathrm{C} 3$ & $1.508(4)$ \\
\hline $\mathrm{K} 1-\mathrm{S} 1^{\mathrm{ii}}$ & $3.3797(14)$ & $\mathrm{C} 4-\mathrm{C} 5$ & $1.508(5)$ \\
\hline $\mathrm{K} 1-\mathrm{S} 2^{\mathrm{ii}}$ & $3.3708(13)$ & $\mathrm{C} 2-\mathrm{H} 2 \mathrm{~A}$ & 0.9700 \\
\hline $\mathrm{K} 1-\mathrm{O} 1^{\mathrm{iii}}$ & $3.007(3)$ & $\mathrm{C} 2-\mathrm{H} 2 \mathrm{~B}$ & 0.9700 \\
\hline $\mathrm{K} 1-\mathrm{O} 2^{\mathrm{iv}}$ & $2.967(3)$ & $\mathrm{C} 3-\mathrm{H} 3 \mathrm{~A}$ & 0.9700 \\
\hline $\mathrm{S} 1-\mathrm{C} 1$ & $1.730(3)$ & $\mathrm{C} 3-\mathrm{H} 3 \mathrm{~B}$ & 0.9700 \\
\hline $\mathrm{S} 2-\mathrm{C} 1$ & $1.707(4)$ & $\mathrm{C} 4-\mathrm{H} 4 \mathrm{~A}$ & 0.9700 \\
\hline $\mathrm{O} 1-\mathrm{C} 3$ & $1.423(4)$ & $\mathrm{C} 4-\mathrm{H} 4 \mathrm{~B}$ & 0.9700 \\
\hline $\mathrm{O} 1-\mathrm{C} 4$ & $1.433(4)$ & $\mathrm{C} 5-\mathrm{H} 5 \mathrm{~A}$ & 0.9700 \\
\hline $\mathrm{O} 2-\mathrm{H} 2 \mathrm{O}$ & $0.85(3)$ & $\mathrm{C} 5-\mathrm{H} 5 \mathrm{~B}$ & 0.9700 \\
\hline $\mathrm{O} 2-\mathrm{H} 1 \mathrm{O}$ & $0.86(4)$ & & \\
\hline $\mathrm{O} 1-\mathrm{K} 1-\mathrm{O} 2$ & $142.34(8)$ & $\mathrm{K} 1^{\mathrm{iv}}-\mathrm{O} 1-\mathrm{C} 3$ & $108.72(17)$ \\
\hline $\mathrm{S} 1 \mathrm{i}-\mathrm{K} 1-\mathrm{O} 1$ & $72.85(5)$ & $\mathrm{K} 1^{\mathrm{iv}}-\mathrm{O} 1-\mathrm{C} 4$ & $110.7(2)$ \\
\hline $\mathrm{S} 2 \mathrm{i}-\mathrm{K} 1-\mathrm{O} 1$ & $99.11(5)$ & $\mathrm{K} 1-\mathrm{O} 2-\mathrm{K} 1^{\mathrm{iii}}$ & $89.96(8)$ \\
\hline $\mathrm{S} 1^{\mathrm{ii}}-\mathrm{K} 1-\mathrm{O} 1$ & $90.44(6)$ & $\mathrm{K} 1^{\mathrm{iii}}-\mathrm{O} 2-\mathrm{H} 2 \mathrm{O}$ & $100(3)$ \\
\hline $\mathrm{S} 2^{\mathrm{ii}}-\mathrm{K} 1-\mathrm{O} 1$ & $89.55(5)$ & $\mathrm{K} 1-\mathrm{O} 2-\mathrm{H} 2 \mathrm{O}$ & $94(3)$ \\
\hline $\mathrm{O} 1-\mathrm{K} 1-\mathrm{O} 1^{\mathrm{iii}}$ & $147.70(8)$ & $\mathrm{H} 1 \mathrm{O}-\mathrm{O} 2-\mathrm{H} 2 \mathrm{O}$ & $112(4)$ \\
\hline $\mathrm{O} 1-\mathrm{K} 1-\mathrm{O} 2^{\mathrm{iv}}$ & $66.04(8)$ & $\mathrm{K} 1-\mathrm{O} 2-\mathrm{H} 1 \mathrm{O}$ & $149(2)$ \\
\hline $\mathrm{S} 1 \mathrm{i}-\mathrm{K} 1-\mathrm{O} 2$ & $142.49(6)$ & 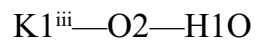 & $101(3)$ \\
\hline $\mathrm{S} 2 \mathrm{i}-\mathrm{K} 1-\mathrm{O} 2$ & $98.33(6)$ & $\mathrm{C} 1-\mathrm{N} 1-\mathrm{C} 5$ & $123.9(3)$ \\
\hline
\end{tabular}




\begin{tabular}{|c|c|c|c|}
\hline $\mathrm{S} 1{ }^{\mathrm{ii}}-\mathrm{K} 1-\mathrm{O} 2$ & $94.95(6)$ & $\mathrm{C} 2-\mathrm{N} 1-\mathrm{C} 5$ & $112.7(2)$ \\
\hline $\mathrm{S} 2 \mathrm{ii}-\mathrm{K} 1-\mathrm{O} 2$ & $65.49(6)$ & $\mathrm{C} 1-\mathrm{N} 1-\mathrm{C} 2$ & $122.6(3)$ \\
\hline $\mathrm{O} 1{ }^{\mathrm{iii}}-\mathrm{K} 1-\mathrm{O} 2$ & $67.68(8)$ & $\mathrm{S} 1-\mathrm{C} 1-\mathrm{N} 1$ & $120.0(3)$ \\
\hline $\mathrm{O} 2-\mathrm{K} 1-\mathrm{O} 2^{\mathrm{iv}}$ & $92.48(9)$ & $\mathrm{S} 2-\mathrm{C} 1-\mathrm{N} 1$ & $120.2(2)$ \\
\hline $\mathrm{S} 1^{\mathrm{i}}-\mathrm{K} 1-\mathrm{S} 2^{\mathrm{i}}$ & $53.28(3)$ & $\mathrm{S} 1-\mathrm{C} 1-\mathrm{S} 2$ & $119.81(16)$ \\
\hline $\mathrm{S} 11^{\mathrm{i}}-\mathrm{K} 1-\mathrm{S} 1^{\mathrm{ii}}$ & $97.59(3)$ & $\mathrm{N} 1-\mathrm{C} 2-\mathrm{C} 3$ & $110.6(3)$ \\
\hline $\mathrm{S} 11^{\mathrm{i}}-\mathrm{K} 1-\mathrm{S} 2^{\mathrm{ii}}$ & $145.67(4)$ & $\mathrm{O} 1-\mathrm{C} 3-\mathrm{C} 2$ & $112.1(3)$ \\
\hline 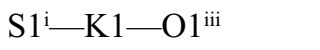 & $83.26(5)$ & $\mathrm{O} 1-\mathrm{C} 4-\mathrm{C} 5$ & $111.7(3)$ \\
\hline $\mathrm{S} 1^{\mathrm{i}}-\mathrm{K} 1-\mathrm{O} 2^{\mathrm{iv}}$ & $94.72(6)$ & $\mathrm{N} 1-\mathrm{C} 5-\mathrm{C} 4$ & $110.7(3)$ \\
\hline $\mathrm{S} 1^{\mathrm{ii}}-\mathrm{K} 1-\mathrm{S} 2^{\mathrm{i}}$ & $143.59(3)$ & $\mathrm{N} 1-\mathrm{C} 2-\mathrm{H} 2 \mathrm{~A}$ & 110.00 \\
\hline $\mathrm{S} 2^{\mathrm{i}}-\mathrm{K} 1-\mathrm{S} 2^{\mathrm{ii}}$ & $161.01(4)$ & $\mathrm{N} 1-\mathrm{C} 2-\mathrm{H} 2 \mathrm{~B}$ & 109.00 \\
\hline $\mathrm{S} 2^{\mathrm{i}}-\mathrm{K} 1-\mathrm{O} 1^{\mathrm{iii}}$ & $82.85(5)$ & $\mathrm{C} 3-\mathrm{C} 2-\mathrm{H} 2 \mathrm{~A}$ & 109.00 \\
\hline $\mathrm{S} 2^{\mathrm{i}}-\mathrm{K} 1-\mathrm{O} 2^{\mathrm{iv}}$ & $64.28(6)$ & $\mathrm{C} 3-\mathrm{C} 2-\mathrm{H} 2 \mathrm{~B}$ & 110.00 \\
\hline $\mathrm{S} 1^{\mathrm{ii}}-\mathrm{K} 1-\mathrm{S} 2^{\mathrm{ii}}$ & $52.27(3)$ & $\mathrm{H} 2 \mathrm{~A}-\mathrm{C} 2-\mathrm{H} 2 \mathrm{~B}$ & 108.00 \\
\hline $\mathrm{S} 1^{\mathrm{ii}}-\mathrm{K} 1-\mathrm{O} 1^{\mathrm{iii}}$ & $71.17(5)$ & $\mathrm{O} 1-\mathrm{C} 3-\mathrm{H} 3 \mathrm{~A}$ & 109.00 \\
\hline $\mathrm{S} 1^{\mathrm{ii}}-\mathrm{K} 1-\mathrm{O} 2^{\mathrm{iv}}$ & $148.77(6)$ & $\mathrm{O} 1-\mathrm{C} 3-\mathrm{H} 3 \mathrm{~B}$ & 109.00 \\
\hline $\mathrm{S} 2^{\mathrm{ii}}-\mathrm{K} 1-\mathrm{O} 1^{\mathrm{iii}}$ & $98.84(5)$ & $\mathrm{C} 2-\mathrm{C} 3-\mathrm{H} 3 \mathrm{~A}$ & 109.00 \\
\hline $\mathrm{S} 2^{\mathrm{ii}}-\mathrm{K} 1-\mathrm{O} 2^{\mathrm{iv}}$ & $104.89(6)$ & $\mathrm{C} 2-\mathrm{C} 3-\mathrm{H} 3 \mathrm{~B}$ & 109.00 \\
\hline $\mathrm{O} 1^{\mathrm{iii}}-\mathrm{K} 1-\mathrm{O} 2^{\mathrm{iv}}$ & $139.03(8)$ & $\mathrm{H} 3 \mathrm{~A}-\mathrm{C} 3-\mathrm{H} 3 \mathrm{~B}$ & 108.00 \\
\hline $\mathrm{K} 1^{\mathrm{v}}-\mathrm{S} 1-\mathrm{C} 1$ & 87.47 (11) & $\mathrm{O} 1-\mathrm{C} 4-\mathrm{H} 4 \mathrm{~A}$ & 109.00 \\
\hline $\mathrm{K} 1{ }^{\mathrm{ii}}-\mathrm{S} 1-\mathrm{C} 1$ & 87.09 (11) & $\mathrm{O} 1-\mathrm{C} 4-\mathrm{H} 4 \mathrm{~B}$ & 109.00 \\
\hline $\mathrm{K} 1^{\mathrm{v}}-\mathrm{S} 1-\mathrm{K} 1^{\mathrm{ii}}$ & 76.09 (3) & $\mathrm{C} 5-\mathrm{C} 4-\mathrm{H} 4 \mathrm{~A}$ & 109.00 \\
\hline $\mathrm{K} 1^{\mathrm{v}}-\mathrm{S} 2-\mathrm{C} 1$ & $84.71(10)$ & $\mathrm{C} 5-\mathrm{C} 4-\mathrm{H} 4 \mathrm{~B}$ & 109.00 \\
\hline $\mathrm{K} 1{ }^{\mathrm{ii}}-\mathrm{S} 2-\mathrm{C} 1$ & $87.73(10)$ & $\mathrm{H} 4 \mathrm{~A}-\mathrm{C} 4-\mathrm{H} 4 \mathrm{~B}$ & 108.00 \\
\hline $\mathrm{K} 1^{\mathrm{v}}-\mathrm{S} 2-\mathrm{K} 1^{\mathrm{ii}}$ & $74.95(2)$ & $\mathrm{N} 1-\mathrm{C} 5-\mathrm{H} 5 \mathrm{~A}$ & 110.00 \\
\hline $\mathrm{K} 1-\mathrm{O} 1-\mathrm{C} 3$ & 120.77 (19) & $\mathrm{N} 1-\mathrm{C} 5-\mathrm{H} 5 \mathrm{~B}$ & 110.00 \\
\hline $\mathrm{K} 1-\mathrm{O} 1-\mathrm{C} 4$ & $118.7(2)$ & $\mathrm{C} 4-\mathrm{C} 5-\mathrm{H} 5 \mathrm{~A}$ & 109.00 \\
\hline $\mathrm{K} 1-\mathrm{O} 1-\mathrm{K} 1^{\mathrm{iv}}$ & $85.98(6)$ & $\mathrm{C} 4-\mathrm{C} 5-\mathrm{H} 5 \mathrm{~B}$ & 109.00 \\
\hline $\mathrm{C} 3-\mathrm{O} 1-\mathrm{C} 4$ & $108.9(2)$ & $\mathrm{H} 5 \mathrm{~A}-\mathrm{C} 5-\mathrm{H} 5 \mathrm{~B}$ & 108.00 \\
\hline $\mathrm{O} 2-\mathrm{K} 1-\mathrm{O} 1-\mathrm{C} 3$ & $142.4(2)$ & $\mathrm{O} 2-\mathrm{K} 1-\mathrm{S} 1^{\mathrm{ii}}-\mathrm{C} 1^{\mathrm{ii}}$ & $-72.98(12)$ \\
\hline $\mathrm{O} 2-\mathrm{K} 1-\mathrm{O} 1-\mathrm{C} 4$ & $3.2(3)$ & $\mathrm{O} 2-\mathrm{K} 1-\mathrm{S} 1^{\mathrm{ii}}-\mathrm{K} 1^{\mathrm{iii}}$ & $15.13(6)$ \\
\hline $\mathrm{O} 2-\mathrm{K} 1-\mathrm{O} 1-\mathrm{K} 1^{\mathrm{iv}}$ & $-108.20(12)$ & $\mathrm{O} 1-\mathrm{K} 1-\mathrm{S} 2^{\mathrm{ii}}-\mathrm{C} 1^{\mathrm{ii}}$ & $-71.26(12)$ \\
\hline $\mathrm{S} 1 \mathrm{i}-\mathrm{K} 1-\mathrm{O} 1-\mathrm{C} 3$ & $-54.13(19)$ & $\mathrm{O} 1-\mathrm{K} 1-\mathrm{S} 2^{\mathrm{ii}}-\mathrm{K} 1^{\mathrm{iii}}$ & $-156.39(6)$ \\
\hline $\mathrm{S} 1 \mathrm{i}-\mathrm{K} 1-\mathrm{O} 1-\mathrm{C} 4$ & $166.7(2)$ & $\mathrm{O} 2-\mathrm{K} 1-\mathrm{S} 2^{\mathrm{ii}}-\mathrm{C} 1^{\mathrm{ii}}$ & $137.86(12)$ \\
\hline $\mathrm{S} 1^{\mathrm{i}}-\mathrm{K} 1-\mathrm{O} 1-\mathrm{K} 1^{\mathrm{iv}}$ & $55.30(5)$ & $\mathrm{O} 2-\mathrm{K} 1-\mathrm{S} 2^{\mathrm{ii}}-\mathrm{K} 1^{\mathrm{iii}}$ & $52.73(7)$ \\
\hline $\mathrm{S} 2 \mathrm{i}-\mathrm{K} 1-\mathrm{O} 1-\mathrm{C} 3$ & $-101.0(2)$ & $\mathrm{O} 1-\mathrm{K} 1-\mathrm{O} 1^{\mathrm{iii}}-\mathrm{K} 1^{\mathrm{iii}}$ & $111.73(12)$ \\
\hline $\mathrm{S} 2 \mathrm{i}-\mathrm{K} 1-\mathrm{O} 1-\mathrm{C} 4$ & $119.8(2)$ & $\mathrm{O} 1-\mathrm{K} 1-\mathrm{O} 1^{\mathrm{iii}}-\mathrm{C} 3^{\mathrm{iii}}$ & $-9.5(2)$ \\
\hline $\mathrm{S} 2^{\mathrm{i}}-\mathrm{K} 1-\mathrm{O} 1-\mathrm{K} 1^{\mathrm{iv}}$ & $8.40(6)$ & $\mathrm{O} 1-\mathrm{K} 1-\mathrm{O} 1^{\mathrm{iii}}-\mathrm{C} 4^{\mathrm{iii}}$ & $-129.1(2)$ \\
\hline $\mathrm{S} 1^{\mathrm{ii}}-\mathrm{K} 1-\mathrm{O} 1-\mathrm{C} 3$ & $43.7(2)$ & $\mathrm{O} 2-\mathrm{K} 1-\mathrm{O} 1^{\mathrm{iii}}-\mathrm{K} 1^{\mathrm{iii}}$ & $-50.45(8)$ \\
\hline $\mathrm{S} 1^{\mathrm{ii}}-\mathrm{K} 1-\mathrm{O} 1-\mathrm{C} 4$ & $-95.5(2)$ & $\mathrm{O} 2-\mathrm{K} 1-\mathrm{O} 1^{\mathrm{iii}}-\mathrm{C} 3^{\mathrm{iii}}$ & $-171.6(2)$ \\
\hline $\mathrm{S} 1^{\mathrm{ii}}-\mathrm{K} 1-\mathrm{O} 1-\mathrm{K} 1^{\mathrm{iv}}$ & $153.11(5)$ & $\mathrm{O} 2-\mathrm{K} 1-\mathrm{O} 1^{\mathrm{iii}}-\mathrm{C} 4^{\mathrm{iii}}$ & $68.8(2)$ \\
\hline $\mathrm{S} 2{ }^{\mathrm{ii}}-\mathrm{K} 1-\mathrm{O} 1-\mathrm{C} 3$ & $96.0(2)$ & $\mathrm{O} 1-\mathrm{K} 1-\mathrm{O} 2^{\mathrm{iv}}-\mathrm{K} 1^{\mathrm{iv}}$ & $52.11(7)$ \\
\hline $\mathrm{S} 2{ }^{\mathrm{ii}}-\mathrm{K} 1-\mathrm{O} 1-\mathrm{C} 4$ & $-43.2(2)$ & $\mathrm{O} 2-\mathrm{K} 1-\mathrm{O} 2^{\mathrm{iv}}-\mathrm{K} 1^{\mathrm{iv}}$ & $-159.92(8)$ \\
\hline 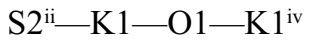 & $-154.62(6)$ & $\mathrm{K} 1 \mathrm{v}-\mathrm{S} 1-\mathrm{C} 1-\mathrm{S} 2$ & $39.50(18)$ \\
\hline 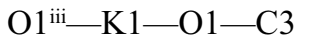 & $-10.0(3)$ & $\mathrm{K} 1 \mathrm{v}-\mathrm{S} 1-\mathrm{C} 1-\mathrm{N} 1$ & $-140.0(2)$ \\
\hline 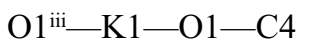 & $-149.2(2)$ & $\mathrm{K} 1{ }^{\mathrm{ii}}-\mathrm{S} 1-\mathrm{C} 1-\mathrm{S} 2$ & $-36.69(18)$ \\
\hline 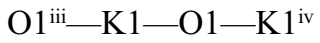 & $99.41(13)$ & $\mathrm{K} 1^{\mathrm{ii}}-\mathrm{S} 1-\mathrm{C} 1-\mathrm{N} 1$ & $143.8(2)$ \\
\hline
\end{tabular}




\begin{tabular}{|c|c|c|c|}
\hline $\mathrm{O} 2^{\mathrm{iv}}-\mathrm{K} 1-\mathrm{O} 1-\mathrm{C} 3$ & $-157.5(2)$ & $\mathrm{K} 11^{\mathrm{v}}-\mathrm{S} 2-\mathrm{C} 1-\mathrm{S} 1$ & $-38.31(18)$ \\
\hline $\mathrm{O} 2^{\mathrm{iv}}-\mathrm{K} 1-\mathrm{O} 1-\mathrm{C} 4$ & $63.4(2)$ & $\mathrm{K} 1{ }^{\mathrm{v}}-\mathrm{S} 2-\mathrm{C} 1-\mathrm{N} 1$ & $141.2(3)$ \\
\hline $\mathrm{O} 2^{\mathrm{iv}}-\mathrm{K} 1-\mathrm{O} 1-\mathrm{K} 1^{\mathrm{iv}}$ & $-48.08(8)$ & $\mathrm{K} 1 \mathrm{ii}-\mathrm{S} 2-\mathrm{C} 1-\mathrm{S} 1$ & $36.78(18)$ \\
\hline $\mathrm{O} 1-\mathrm{K} 1-\mathrm{O} 2-\mathrm{K} 1^{\mathrm{iii}}$ & $-113.36(12)$ & $\mathrm{K} 1 \mathrm{ii}-\mathrm{S} 2-\mathrm{C} 1-\mathrm{N} 1$ & $-143.7(2)$ \\
\hline 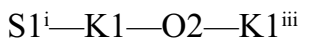 & $93.11(11)$ & $\mathrm{K} 1-\mathrm{O} 1-\mathrm{C} 3-\mathrm{C} 2$ & $-81.8(3)$ \\
\hline $\mathrm{S} 2^{\mathrm{i}}-\mathrm{K} 1-\mathrm{O} 2-\mathrm{K} 1^{\mathrm{iii}}$ & $129.81(6)$ & $\mathrm{C} 4-\mathrm{O} 1-\mathrm{C} 3-\mathrm{C} 2$ & $60.9(3)$ \\
\hline 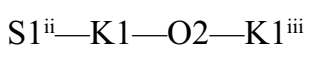 & $-16.20(6)$ & $\mathrm{K} 11^{\mathrm{iv}}-\mathrm{O} 1-\mathrm{C} 3-\mathrm{C} 2$ & $-178.43(19)$ \\
\hline $\mathrm{S} 2^{\mathrm{ii}}-\mathrm{K} 1-\mathrm{O} 2-\mathrm{K} 1^{\mathrm{iii}}$ & $-60.59(5)$ & $\mathrm{K} 1-\mathrm{O} 1-\mathrm{C} 4-\mathrm{C} 5$ & $82.8(3)$ \\
\hline 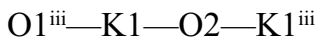 & $51.11(7)$ & $\mathrm{C} 3-\mathrm{O} 1-\mathrm{C} 4-\mathrm{C} 5$ & $-60.7(3)$ \\
\hline $\mathrm{O} 2^{\mathrm{iv}}-\mathrm{K} 1-\mathrm{O} 2-\mathrm{K} 1^{\mathrm{iii}}$ & $-165.83(8)$ & $\mathrm{K} 11^{\mathrm{iv}}-\mathrm{O} 1-\mathrm{C} 4-\mathrm{C} 5$ & $179.8(2)$ \\
\hline $\mathrm{O} 1-\mathrm{K} 1-\mathrm{S} 1^{\mathrm{i}}-\mathrm{C}^{\mathrm{i}}$ & $-136.36(13)$ & $\mathrm{C} 2-\mathrm{N} 1-\mathrm{C} 1-\mathrm{S} 1$ & $-176.4(2)$ \\
\hline $\mathrm{O} 1-\mathrm{K} 1-\mathrm{S} 1^{\mathrm{i}-}-\mathrm{K} 1^{\mathrm{iv}}$ & $-48.73(6)$ & $\mathrm{C} 2-\mathrm{N} 1-\mathrm{C} 1-\mathrm{S} 2$ & $4.1(4)$ \\
\hline $\mathrm{O} 2-\mathrm{K} 1-\mathrm{S} 1^{\mathrm{i}}-\mathrm{Cl}^{\mathrm{i}}$ & $27.08(16)$ & $\mathrm{C} 5-\mathrm{N} 1-\mathrm{C} 1-\mathrm{S} 1$ & $-8.1(4)$ \\
\hline $\mathrm{O} 2-\mathrm{K} 1-\mathrm{S} 1^{\mathrm{i}}-\mathrm{K} 1^{\mathrm{iv}}$ & $114.71(11)$ & $\mathrm{C} 5-\mathrm{N} 1-\mathrm{C} 1-\mathrm{S} 2$ & $172.4(2)$ \\
\hline $\mathrm{O} 1-\mathrm{K} 1-\mathrm{S} 2^{\mathrm{i}}-\mathrm{C} 1^{\mathrm{i}}$ & $81.34(12)$ & $\mathrm{C} 1-\mathrm{N} 1-\mathrm{C} 2-\mathrm{C} 3$ & $-140.7(3)$ \\
\hline $\mathrm{O} 1-\mathrm{K} 1-\mathrm{S} 2^{\mathrm{i}}-\mathrm{K} 1^{\mathrm{iv}}$ & $-7.73(6)$ & $\mathrm{C} 5-\mathrm{N} 1-\mathrm{C} 2-\mathrm{C} 3$ & $49.8(3)$ \\
\hline $\mathrm{O} 2-\mathrm{K} 1-\mathrm{S} 22^{\mathrm{i}}-\mathrm{C} 1^{\mathrm{i}}$ & $-132.18(12)$ & $\mathrm{C} 1-\mathrm{N} 1-\mathrm{C} 5-\mathrm{C} 4$ & $140.7(3)$ \\
\hline $\mathrm{O} 2-\mathrm{K} 1-\mathrm{S} 22^{\mathrm{i}}-\mathrm{K} 1^{\mathrm{iv}}$ & $138.75(7)$ & $\mathrm{C} 2-\mathrm{N} 1-\mathrm{C} 5-\mathrm{C} 4$ & $-50.0(3)$ \\
\hline $\mathrm{O} 1-\mathrm{K} 1-\mathrm{S} 1^{\mathrm{ii}}-\mathrm{C} 1^{\mathrm{ii}}$ & $69.70(12)$ & $\mathrm{N} 1-\mathrm{C} 2-\mathrm{C} 3-\mathrm{O} 1$ & $-55.7(3)$ \\
\hline $\mathrm{O} 1-\mathrm{K} 1-\mathrm{S} 1^{\mathrm{ii}}-\mathrm{K} 1^{\mathrm{iii}}$ & $157.81(5)$ & $\mathrm{O} 1-\mathrm{C} 4-\mathrm{C} 5-\mathrm{N} 1$ & $55.5(3)$ \\
\hline
\end{tabular}

Symmetry codes: (i) $-x, y+1 / 2,-z+3 / 2$; (ii) $-x,-y,-z+1$; (iii) $x,-y+1 / 2, z-1 / 2$; (iv) $x,-y+1 / 2, z+1 / 2$; (v) $-x, y-1 / 2,-z+3 / 2$.

Hydrogen-bond geometry $\left(A,{ }^{\circ}\right)$

\begin{tabular}{lllll}
\hline$D-\mathrm{H} \cdots A$ & $D-\mathrm{H}$ & $\mathrm{H} \cdots A$ & $D \cdots A$ & $D-\mathrm{H} \cdots A$ \\
\hline $\mathrm{O} 2-\mathrm{H} 1 O \cdots \mathrm{S} 1^{\text {vi }}$ & $0.86(4)$ & $2.45(4)$ & $3.219(3)$ & $149(3)$ \\
$\mathrm{O} 2-\mathrm{H} 2 O \cdots 1^{\text {vii }}$ & $0.85(3)$ & $2.87(5)$ & $3.462(3)$ & $129(4)$
\end{tabular}

Symmetry codes: (vi) $-x-1, y+1 / 2,-z+1 / 2$; (vii) $-x-1,-y,-z+1$. 